\title{
Crops of Papules and Papulonodular Plaques- An Unusual Presentation of Cutaneous Metastasis- A Case Report
}

\section{Sohail Chaudhry Z*, Ghias A, Asghar A and Jameel Ahmad T}

Department of dermatology, mayo hospital, Pakistan

*Corresponding author: Dr. Zaima Sohail Chaudhry, FCPS-II Dermatology(fellowship), Department of Dermatology Unit II, King Edward Medical University/ Mayo Hospital Lahore, Pakistan, Email: zaeema.chaudhry7@gmail.com

\section{Case Report}

Volume 5 Issue 4

Received Date: September 23, 2020

Published Date: October 16, 2020

DOI: $10.23880 /$ cdoaj-16000221

\section{Abstract}

Cutaneous Metastasis refers to a growth of neoplastic cells in the skin originating from an internal malignancy. Morphologically the metastasis could take multiple morphologies. We report a case of cutaneous metastasis in a female of age $34 y e a r s$. She initially presented with a 2 years history of progressive appearance of multiple asymptomatic clusters of monomorphic papulonodular lesions widespread on her trunk, lower limbs and genitalia later developed dyspnoea and weight loss. Chest CT showed moderate pleural effusion and multiple lymphadenopathies and right sided lung mass/consolidation. Histopathological evidences of cutaneous biopsy revealed cutaneous Metastasis consistent with the lung primary.

Keywords: Malignant epithelial neoplasm; Metastasis; Papulonodules

\section{Introduction}

Cutaneous metastasis have a variable clinical appearances and can even mimic benign skin lesions. Skin is regarded as an uncommon metastatic site for internal malignancies however when present, they are usually diagnosed after the primary disease has been identified [1].

Sometimes they can be the only presenting lesion as well. The recognition of cutaneous metastasis often dramatically alters the therapeutic plan, especially when the metastasis signify cancer originally thought to be cured. In a review by Looking Bill,, et al. including 7316 patients, skin involvement was seen in only 0.8 percent [2] Being first sign of presentation, cutaneous metastases are biopsied to locate the primary cancer. Among the Cutaneous Metastatic cancers Lung is the most common primary site of underlying malignancy in men (24\%) and 4th common primary site in women (4\%) [1]. The presence of cutaneous metastasis in lung cancer portends a poor prognosis, with an average survival ranging between 3 to 5 months in majority of the studies [3-5]. Metastatic skin lesions usually present as carcinoma erysipeloides, sclerodermoid/ en cuirasse or carcinoma talengiectoides but papillomatosis plaques or clusters of papulonodular lesions or molluscum contagiosum like lesions are a rare entity. In this report we describe an unusual case of lung adenocarcinoma metastasizing to skin. Metastasis in skin can be followed by multiple internal lymphadenopathies, subcutaneous, intestinal, pelvic and bony metastasis (L 3-5, T 6-T12)

\section{Case Report}

A 34 years old female, mother of 2 children presented with a two years history of progressive appearance of multiple asymptomatic crops of monomorphic papulonodular skin lesions widespread on her trunk (chest, abdomen, back) and also on the lower limbs and genitalia (vulva and perianal fold) sparing her upper limbs and face.

There were areas of discrete warty papules and papillomatous plaques of various sizes. The nodules were exuberant, firm to hard, some being skin coloured and the rest with fleshy violacious hue, arranged in numerous groups. There were ulcerovegetant tumorous lesions more 
prominent on the right breast, clinically measuring 3 to $4 \mathrm{~cm}$, indurated and infiltrated. The right nipple was distorted however the left was apparently normal. There was evidence of superimposed bacterial skin infection apparent on her back. There were palpable subcutaneous nodules on her right breast and left shoulder, the largest measuring 3-4 in diameter. A group of discrete posterior cervical lymph nodes was palpable measuring $1 \mathrm{~cm}$ in diameter approximately, however they were mobile/ not adherent to the underlying tissue, firm yet non-tender. Her lower limbs were markedly edematous with profound vulvar swelling. The cachexia was marked too.

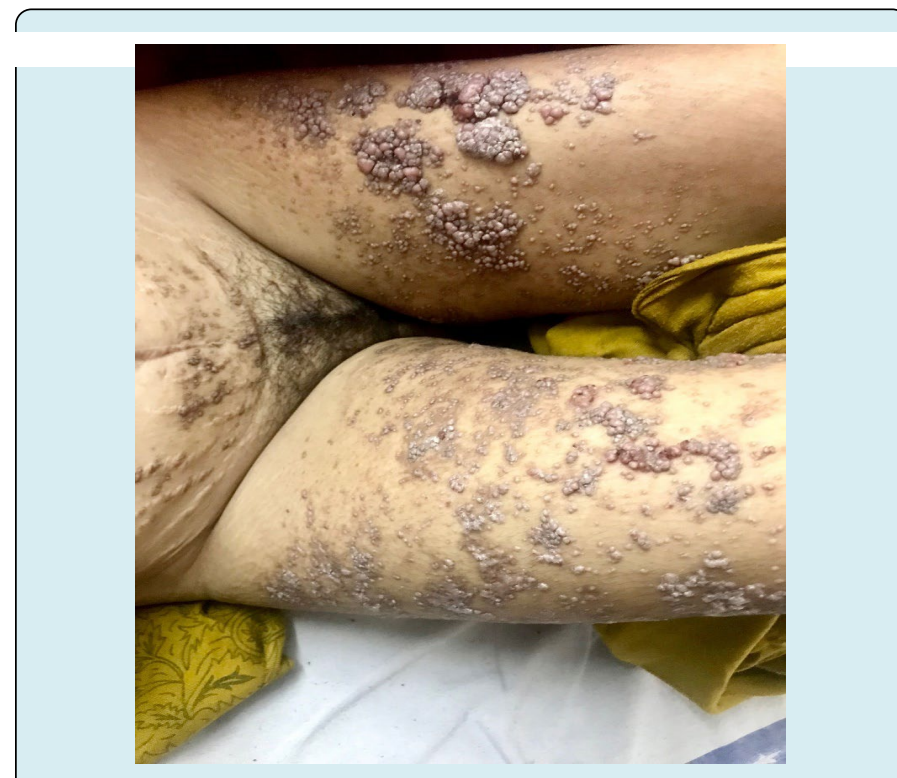

Figure 1: Exuberant violaceous papulomatous plaques present bilaterally on legs.

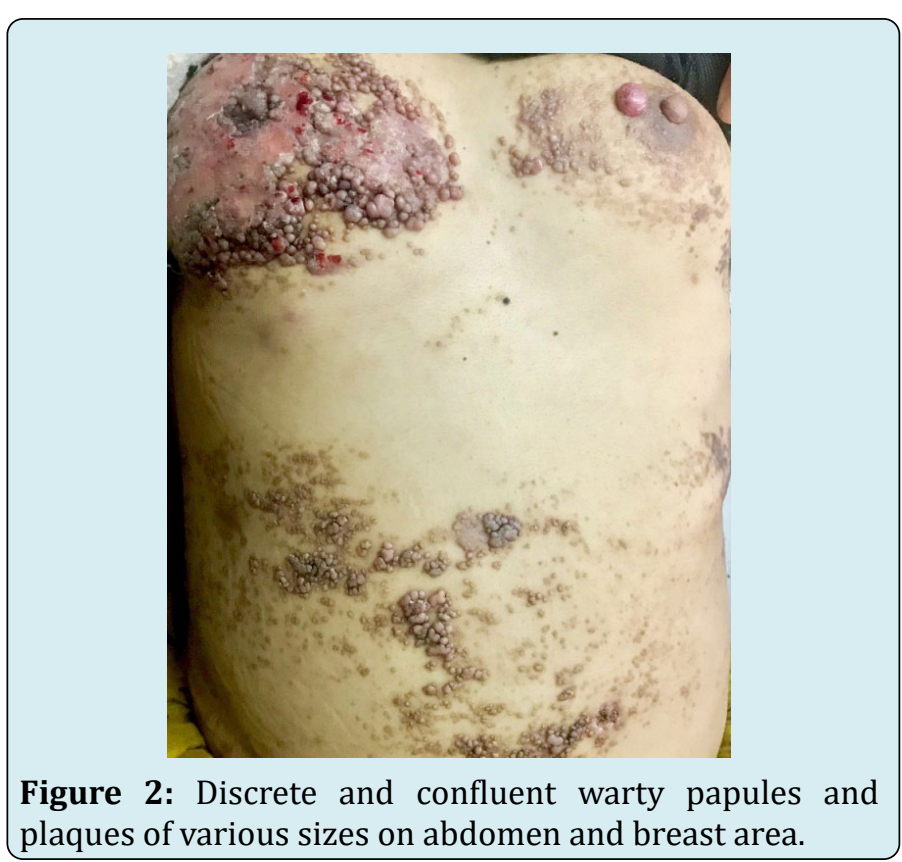

Histopathological findings from two of the papules was consistent with Metastatic Adenocarcinoma.

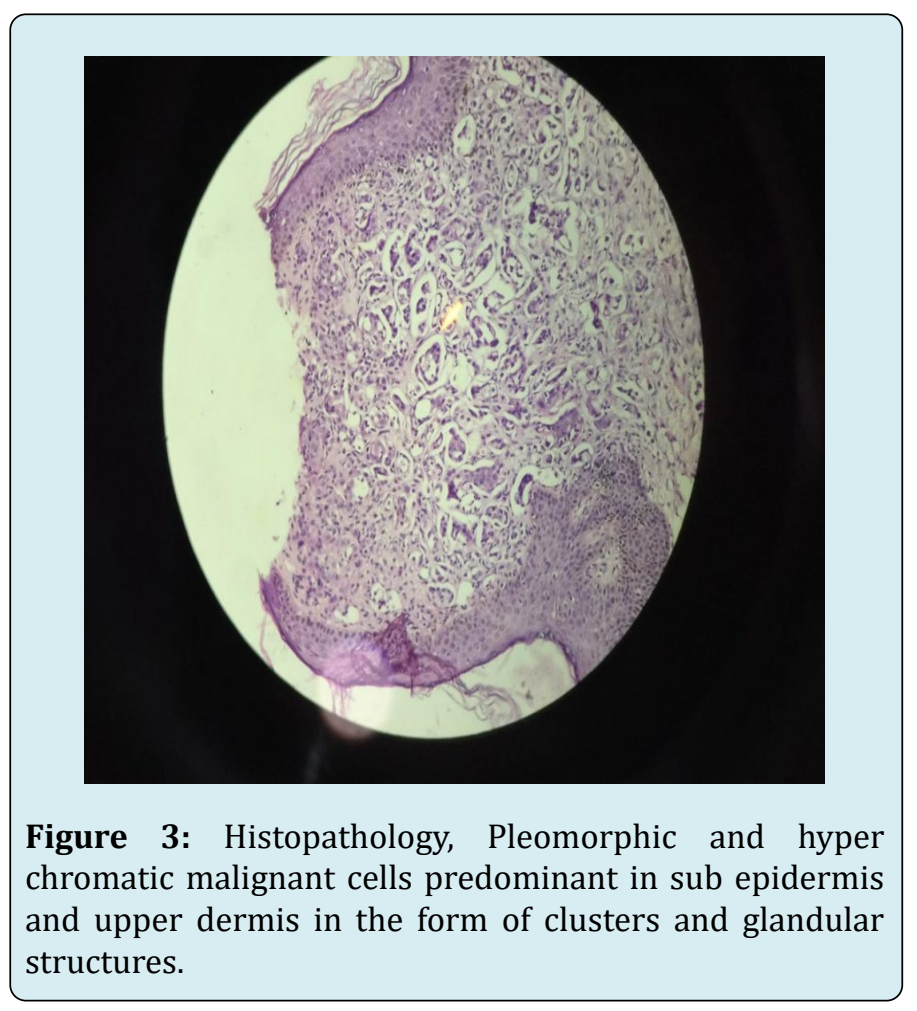

There was positive TTF-1(thyroid transcription factor) expression. It is present uniformly in the terminal respiratory unit, which comprises peripheral airway cells and small-sized bronchioles and plays a crucial role in normal lung function and morphogenesis. In order to locate for the primary tumour, multiple laboratory investigations were carried out.

Bilateral breast ultrasound was unremarkable yet CT-chest showed moderate pleural effusion, hilar and mediastinal lymphadenopathy and an area of consolidation/ mass on right. Pleural fluid aspiration was performed under ultrasound guidance and subsequent cytology was suggestive of malignant effusion. Abdominal CT-scan revealed no organ enlargements but inguinal and para-aortic lymphadenopathy was noted. Metastatic nodules were also observed in the abdomen, and pelvis and bony metastasis at $\mathrm{L} 1$ and L4 and T6 - T 12. CEA levels were markedly raised however CA 125 and CA 15.5 were unremarkable. Complete blood examination revealed bicytopenia with neutrophilic lymphocytosis. Serum uric acid level was also raised and rest of labs were within normal range.

\section{Discussion}

Cutaneous metastases are encountered in $0.7-9 \%$ of all patients with internal malignancy despite the fact that skin 


\section{Clinical Dermatology Open Access Journal}

is an uncommon site of metastatic disease when compared to other organs [6]. Dissemination of visceral malignancies to the skin is rather rare and usually occurs in a later stage of the disease. However, cutaneous metastases may be the first indication of the clinically silent visceral malignancies. In case of lung cancer, metastasis to the skin is much less frequent than that to other organs (brain, bone, liver and adrenal glands). A meta-analysis conducted at Houston, USA in 2003 showed the incidence of cutaneous metastases in lung cancer was $3.4 \%$ in 89 patients among 2,597 subjects [7]. A retrospective study in 2012 in China indicated that $2.8 \%$ of 2,130 patients with advanced non-small cell lung cancer (NSCLC) showed cutaneous metastases as an initial presentation [3]. Clinically, lung cancer may be initially detected by cutaneous metastasis, since the primary lung lesion often remains quiescent, as in our case.

Cutaneous metastasis can manifest as a nodule, ulceration, cellulitis-like lesion, bullae or fibrotic process. Generally, the nodular type is the result of hematogenous metastasis, and is likely to be the most common. Nodules are painless, mobile or fixed, firm or rubbery, discrete or multiple. They vary in color from flesh tones to red-purple, or blue-black and vary in diameter from $5 \mathrm{~mm}$ to $6 \mathrm{~cm}$ [8]. Multiple lesions are usually grouped. They initially grow rapidly, and then more slowly, and may necrotize or ulcerate [9]. The regional distribution of the cutaneous metastasis, although not always predictable, is related to the location of the primary malignancy and the mechanism of metastatic spread. The relative frequency of cutaneous metastasis correlates with the type of primary cancer, which occurs in each sex. For instance, lung and breast carcinomas are the most common primaries that send cutaneous metastasis in men and women, respectively. The head and neck region and the anterior chest are the areas of greatest predilection in men. The anterior chest wall and the abdomen are the most commonly involved sites in women [6]. While certain cancer types are characterized by random distribution for cutaneous metastasis (liver cancer), a number of cancers demonstrate a colonization preference to the region of origin: lung cancer to the supra-diaphragmatic (mostly chest) and colorectal cancers to the infra-diaphragmatic (abdominal) skin regions. In certain cases, however, cutaneous metastasis develops more frequently at specific distant locations, as evidenced by the dissemination of renal cancer to the head and neck region [10]. These findings are clinically relevant and useful especially in patients where cutaneous metastasis is the first indication of a malignancy [11-16].

When approached broadly, one retrospective study of 4,020 patients showed that breast, melanoma, and lung, in that order, top the list for most common cancers to spread to the skin [17]. For older men who present with skin metastases, lung cancer is the most common primary, at about $24 \%$, followed by colorectal cancer, melanoma, and carcinoma of the oral cavity. In women, lung cancer ranks fourth after primary breast cancer, colon cancer, and melanoma [16,17]. Overall, if a patient has lung cancer, their chance of cutaneous metastasis varies, ranging $1 \%$ to $12 \%$. Although the skin is not the first organ it usually spreads to, when it does it does so quickly, with mean time of less than six months [17].

The cutaneous metastasis observed in our case were located on the chest, abdomen, back, lower limbs and genitalia. These were monomorphic, grouped papulonodular type and grew rapidly to an unusual size associated with ulceration.

Physically, cutaneous metastatic lesions due to lung cancer are indistinguishable from those due to carcinoma originating elsewhere in the body. A strong and diffuse nuclear expression of TTF- 1 immunostain is characteristic of primary lung cancer and thyroid carcinoma, which is helpful for identifying the histological origin of primary cancer. Mutually exclusive to EGFR and $A L K$ mutations, KRAS mutants are detected mainly in adenocarcinomas, and show association with progressive disease status. Given the positive expression of TTF-1 in our patient's cutaneous metastasis, we further confirmed the primary malignancy as lung adenocarcinoma. However, thus far the relationship between the histological type of lung cancer and the occurrence of cutaneous metastasis has not been well-defined. Schoenlaub, et al. [11] demonstrated that lung large cell carcinoma had the greatest tendency to metastasize to the skin and lung squamous cell carcinoma the least. On the contrary, lung adenocarcinoma had the greatest tendency and lung large cell carcinoma the least in Hidaka's report [12]. So far, we have not found any reports regarding gene mutation analysis in lung cancer patients with cutaneous metastatic lesions except for one case report to show that KRAS-mutant lung adenocarcinoma can be associated with cutaneous metastasis.

Patients with a lung cancer and cutaneous metastasis are not likely to be cured. Such metastasis signifies a fast-growing aggressive primary lung carcinoma. These patients have an extremely poor prognosis with an average survival ranging between three and five months in the majority of studies [3-5]. Generally, therefore, only palliative chemotherapy is offered. Radiation therapy to the cutaneous metastasis is indicated if associated with severe pain or bleeding. Tyrosine kinase inhibitors (TKIs) have been in use as cancer therapeutics for nearly a decade, and their utility in targeting specific malignancies with defined genetic lesions has proven to be remarkably effective. Recent efforts to characterize the spectrum of genetic lesions found in NSCLC have provided important insights into the molecular basis of this disease and have also revealed a wide array of tyrosine 
kinases that might be effectively targeted for rationally designed therapies. Even though KRAS mutations were identified in NSCLC tumors more than 20 years ago, these tend to occur more frequently in lung adenocarcinomas (approximately 30\%) and less frequently in the squamous cell carcinomas (approximately 5\%) [13]. KRAS mutation is a poor prognostic factor. Recent studies indicate that patients with mutant KRAS tumors fail to benefit from adjuvant chemotherapy and do not respond to EGFR inhibitors [13]. Our patient was put on chemotherapy with carboplatin and paclitaxel. So far her chemotherapy session is not suggestive of any clinical improvement however the supportive therapy has made her drastic improvement in her symptomatology.

\section{Conclusion}

We should be familiar with the predominant morphological features of cutaneous metastasis and should be vigilant enough to account for their rapid course of cutaneous manifestations as reported in our patient and any patient presenting with these lesions with or without systemic upsets should raise our index of suspicion to further investigate the patient for internal malignancies. More over Lung adenocarcinoma are thought to respond poorly to targeted therapies with a poor survival rate.

\section{References}

1. Mollet TW, Garcia CA, Koester G (2009) Skin metastases from lung cancer. Dermatol Online J 15: 1.

2. Lookingbill DP, Spangler N, Sexton FM (1990) Skin involvement as the presenting sign of internal carcinoma. A retrospective study of 7316 cancer patients. J Am Acad Dermatol 22: 19-26.

3. Song Z, Lin B, Shao L, Yiping Z (2012) Cutaneous metastasis as an initial presentation in advanced nonsmall cell lung cancer and its poor survival prognosis. J Cancer Res Clin Oncol 138: 1613-1617.

4. Giroux Leprieur E, Lavole A, Ruppert AM, Valérie G, Marie W, et al. (2012) Factors associated with long-term survival of patients with advanced non-small cell lung cancer. Respirology 17(1): 134-142.

5. Sörenson S, Glimelius B, Nygren P (2001) A systematic overview of chemotherapy effects in non-small cell lung cancer. Acta Oncol 40(3): 327-339.

6. Hussein MR (2010) Skin metastasis: a pathologist's perspective. J Cutan Pathol 37(9): 1-20.

7. Krathen RA, Orengo IF, Rosen T (2003) Cutaneous metastasis: a meta-analysis of data. South Med J 96(2): 164-167.

8. Dreizen S, Dhingra HM, Chiuten DF, Umsawasdi T, Valdivieso M (1986) Cutaneous and subcutaneous metastases of lung cancer. Clinical characteristics. Postgrad Med 80(8): 111-116.

9. Vila JM, Casanova Seuma JM, Portero L (1994) Skin metastasis as the $1^{\text {st }}$ manifestation of bronchogenic carcinoma. Arch Bronconeumol 30: 314-316.

10. Kovács KA, Hegedus B, Kenessey I (2013) Tumor type-specific and skin region-selective metastasis of human cancers: another example of the "seed and soil" hypothesis. Cancer Metastasis Rev 32: 493-499.

11. Schoenlaub P, Sarraux A, Grosshans E (2001) Survival after cutaneous metastasis: a study of 200 cases. Ann Dermatol Venereol 128: 1310-1315.

12. Hidaka T, Ishii Y, Kitamura S (1996) Clinical features of skin metastasis from lung cancer. Intern Med 35: 459462.

13. Roberts PJ, Stinchcombe TE, Der CJ (2010) Personalized medicine in non-small-cell lung cancer: is KRAS a useful marker in selecting patients for epidermal growth factor receptor-targeted therapy? J Clin Oncol 28(31): 47694777.

14. Douillard JY, Shepherd FA, Hirsh V (2010) Molecular predictors of outcome with gefitinib and docetaxel in previously treated non-small-cell lung cancer: data from the randomized phase III INTEREST trial. J Clin Oncol 28(5): 744-752.

15. Lynch TJ, Bell DW, Sordella R, Gurubhagavatula S, Okimoto RA, et al. (2004) Activating mutations in the epidermal growth factor receptor underlying responsiveness of non-small-cell lung cancer to gefitinib. N Engl J Med 350: 2129-2139.

16. Mollet TW, Garcia CA, Koester G (2009) Skin metastases from lung cancer. Dermatol Online J 15(5).

17. Lookingbill DP, Spangler N, Helm KF (1993) Cutaneous metastases in patients with metastatic carcinoma: a retrospective study of 4020 patients. J Am Acad Dermatol 29(2): 228-236.

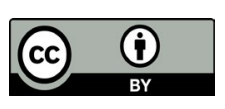

Sohail Chaudhry Z, et al. Crops of Papules and Papulonodular Plaques- An Unusual Presentation of Cutaneous Metastasis- A Case Report. Clin Dermatol J 2020, 5(4): 000221. 